PROCEEDINGS OF THE

AMERICAN MATHEMATICAL SOCIETY

Volume 131, Number 10, Pages 3129-3134

S 0002-9939(03)06986-7

Article electronically published on February 28, 2003

\title{
EXTREMAL PROPERTIES OF THE DERIVATIVES OF THE NEWMAN POLYNOMIALS
}

\author{
TAMÁS ERDÉLYI
}

(Communicated by Jonathan M. Borwein)

\begin{abstract}
Let $\Lambda_{n-1}:=\left\{\lambda_{1}, \lambda_{2}, \ldots, \lambda_{n}\right\}$ be a set of $n$ distinct positive numbers. The span of

$$
\left\{e^{-\lambda_{1} t}, e^{-\lambda_{2} t}, \ldots, e^{-\lambda_{n} t}\right\}
$$

over $\mathbb{R}$ will be denoted by

$$
E\left(\Lambda_{n-1}\right):=\operatorname{span}\left\{e^{-\lambda_{1} t}, e^{-\lambda_{2} t}, \ldots, e^{-\lambda_{n} t}\right\} .
$$
\end{abstract}

Our main result of this note is the following.

Theorem. Suppose $0<q \leq p \leq \infty$. Let $\mu$ be a non-negative integer. Then there are constants $c_{1}(p, q, \mu)>0$ and $c_{2}(p, q, \mu)>0$ depending only on $p, q$, and $\mu$ such that

$$
\begin{aligned}
& c_{1}(p, q, \mu)\left(\sum_{j=1}^{n} \lambda_{j}\right)^{\mu+\frac{1}{q}-\frac{1}{p}} \\
& \leq \sup _{Q \in E\left(\Lambda_{n-1}\right)} \frac{\left\|Q^{(\mu)}\right\|_{L_{p}[0, \infty)}}{\|Q\|_{L_{q}[0, \infty)}} \leq c_{2}(p, q, \mu)\left(\sum_{j=1}^{n} \lambda_{j}\right)^{\mu+\frac{1}{q}-\frac{1}{p}},
\end{aligned}
$$

where the lower bound holds for all $0<q \leq p \leq \infty$ and for all $\mu \geq 0$, while the upper bound holds when $\mu=0$ and $0<q \leq p \leq \infty$ and when $\mu \geq 1, p \geq 1$, and $0<q \leq p \leq \infty$.

\section{INTRODUCTION AND NOTATION}

Let $\Lambda_{n}:=\left\{\lambda_{0}, \lambda_{1}, \ldots, \lambda_{n}\right\}$ be a set of $n+1$ distinct non-negative numbers. The span of

$$
\left\{x^{\lambda_{0}}, x^{\lambda_{1}}, \ldots, x^{\lambda_{n}}\right\}
$$

over $\mathbb{R}$ will be denoted by

$$
M\left(\Lambda_{n}\right):=\operatorname{span}\left\{x^{\lambda_{0}}, x^{\lambda_{1}}, \ldots, x^{\lambda_{n}}\right\} .
$$

Elements of $M\left(\Lambda_{n}\right)$ are called Müntz polynomials of $n+1$ terms. The span of

$$
\left\{e^{-\lambda_{0} t}, e^{-\lambda_{1} t}, \ldots, e^{-\lambda_{n} t}\right\}
$$

Received by the editors April 29, 2002.

2000 Mathematics Subject Classification. Primary 41A17; Secondary 30B10, 26D15.

Key words and phrases. Müntz polynomials, exponential sums, Markov-type inequality, Nikolskii-type inequality, Newman's inequality.

This research was supported, in part, by the NSF under Grant No. DMS-0070826. 
over $\mathbb{R}$ will be denoted by

$$
E\left(\Lambda_{n}\right):=\operatorname{span}\left\{e^{-\lambda_{0} t}, e^{-\lambda_{1} t}, \ldots, e^{-\lambda_{n} t}\right\} .
$$

Elements of $E\left(\Lambda_{n}\right)$ are called exponential sums of $n+1$ terms.

For a function $f$ defined on a set $A$ let

$$
\|f\|_{A}:=\sup \{|f(x)|: x \in A\},
$$

and let

$$
\|f\|_{L_{p} A}:=\left(\int_{A}|f(x)|^{p} d x\right)^{1 / p}, \quad p>0,
$$

whenever the Lebesgue integral exists. Newman's beautiful inequality (see 11 and [4]) is an essentially sharp Markov-type inequality for $M\left(\Lambda_{n}\right)$ on $[0,1]$ in the case when each $\lambda_{j}$ is non-negative.

Theorem 1.1 (Newman's inequality). Let $\Lambda_{n}:=\left\{\lambda_{0}, \lambda_{1}, \ldots, \lambda_{n}\right\}$ be a set of $n+1$ distinct non-negative numbers. Then

$$
\frac{2}{3} \sum_{j=0}^{n} \lambda_{j} \leq \sup _{0 \neq Q \in M\left(\Lambda_{n}\right)} \frac{\left\|x Q^{\prime}(x)\right\|_{[0,1]}}{\|Q\|_{[0,1]}} \leq 9 \sum_{j=0}^{n} \lambda_{j}
$$

or equivalently

$$
\frac{2}{3} \sum_{j=0}^{n} \lambda_{j} \leq \sup _{0 \neq Q \in E\left(\Lambda_{n}\right)} \frac{\left\|Q^{\prime}\right\|_{[0, \infty]}}{\|Q\|_{[0, \infty]}} \leq 9 \sum_{j=0}^{n} \lambda_{j} .
$$

An $L_{p}$ version of this is established in [1], [2], and [3].

Theorem 1.2. Let $1 \leq p \leq \infty$. Let $\Lambda_{n}:=\left\{\lambda_{0}, \lambda_{1}, \ldots, \lambda_{n}\right\}$ be a set of $n+1$ distinct real numbers greater than $-1 / p$. Then

$$
\left\|x Q^{\prime}(x)\right\|_{L_{p}[0,1]} \leq\left(1 / p+9\left(\sum_{j=0}^{n}\left(\lambda_{j}+1 / p\right)\right)\right)\|Q\|_{L_{p}[0,1]}
$$

for every $Q \in M\left(\Lambda_{n}\right)$. This follows from the fact that if $\Lambda_{n}:=\left\{\lambda_{0}, \lambda_{1}, \ldots, \lambda_{n}\right\}$ is a set of $n+1$ distinct non-negative numbers, then

$$
\left\|Q^{\prime}\right\|_{L_{p}[0, \infty)} \leq 9\left(\sum_{j=0}^{n} \gamma_{j}\right)\|Q\|_{L_{p}[0, \infty)}
$$

for every $Q \in E\left(\Lambda_{n}\right)$.

A simple consequence of Theorem 1.1 is the following.

Theorem 1.3 (Nikolskii-type inequality). Suppose $0<q \leq p \leq \infty$. Let $\Lambda_{n}:=$ $\left\{\lambda_{0}, \lambda_{1}, \ldots, \lambda_{n}\right\}$ be a set of $n+1$ distinct real numbers greater than $-1 / q$. Then

$$
\left\|x^{1 / q-1 / p} Q(x)\right\|_{L_{p}[0,1]} \leq c(p, q)\left(\sum_{j=0}^{n}\left(\lambda_{j}+1 / q\right)\right)^{1 / q-1 / p}\|Q\|_{L_{q}[0,1]}
$$

for every $Q \in M\left(\Lambda_{n}\right)$. Equivalently, if $\Lambda_{n}:=\left\{\lambda_{0}, \lambda_{1}, \ldots, \lambda_{n}\right\}$ is a set of $n+1$ distinct non-negative numbers, then

$$
\|Q\|_{L_{p}[0, \infty)} \leq c(p, q)\left(\sum_{j=0}^{n} \lambda_{j}\right)^{1 / q-1 / p}\|Q\|_{L_{q}[0, \infty)}
$$


for every $Q \in E\left(\Lambda_{n}\right)$. In both inequalities

$$
c(p, q):=\left(18 \cdot 2^{q}\right)^{1 / q-1 / p}
$$

is a suitable choice.

The purpose of this note is to show that both Theorems 1.2 and 1.3 are essentially sharp.

\section{NEW RESUlts}

The upper bound in our main theorem below follows as a combination of Theorems 1.2 ans 1.3. The novelty of this note is the establishment of the lower bound.

Theorem 2.1. Let $\Lambda_{n-1}:=\left\{\lambda_{1}, \lambda_{2}, \ldots, \lambda_{n}\right\}$ be a set of $n$ distinct positive numbers. Suppose $0<q \leq p \leq \infty$. Let $\mu$ be a non-negative integer. Then there are constants $c_{1}(p, q, \mu)>0$ and $c_{2}(p, q, \mu)>0$ depending only on $p, q$, and $\mu$ such that

$$
\begin{aligned}
c_{1}(p, q, \mu)\left(\sum_{j=1}^{n} \lambda_{j}\right)^{\mu+\frac{1}{q}-\frac{1}{p}} & \leq \sup _{Q \in E\left(\Lambda_{n-1}\right)} \frac{\left\|Q^{(\mu)}\right\|_{L_{p}[0, \infty)}}{\|Q\|_{L_{q}[0, \infty)}} \\
& \leq c_{2}(p, q, \mu)\left(\sum_{j=1}^{n} \lambda_{j}\right)^{\mu+\frac{1}{q}-\frac{1}{p}},
\end{aligned}
$$

where the lower bound holds for all $0<q \leq p \leq \infty$ and for all $\mu \geq 0$, while the upper bound holds when $\mu=0$ and $0<q \leq p \leq \infty$ and when $\mu \geq 1, p \geq 1$, and $0<q \leq p \leq \infty$.

\section{Proofs}

Proof of Theorem 2.1. As we have already remarked, we need to prove only the lower bound. To this end without loss of generality we may assume that the elements of $\Lambda_{n-1}$ satisfy $\sum_{j=1}^{n} \lambda_{j}=1$; the general result follows by a linear scaling. Then the Newman "polynomial" $T_{n} \in E\left(\Lambda_{n-1}\right)$ is defined by

$$
T_{n}(t):=\frac{1}{2 \pi i} \int_{\Gamma} \frac{e^{-z t}}{B_{n}(z)} d z
$$

where

$$
\Gamma:=\{z \in \mathbb{C}:|z-1|=1\} \quad \text { and } \quad B_{n}(z):=\prod_{j=1}^{n} \frac{z-\lambda_{j}}{z+\lambda_{j}} .
$$

By the residue theorem

$$
T_{n}(t)=\sum_{j=1}^{n}\left(B_{n}^{\prime}\left(\lambda_{j}\right)\right)^{-1} e^{-\lambda_{j} t}
$$

and hence $T_{n} \in E\left(\Lambda_{n-1}\right)$. We claim that

$$
\left|B_{n}(z)\right| \geq \frac{1}{3}, \quad z \in \Gamma .
$$

Indeed, it is easy to see that $0 \leq \lambda_{j} \leq 1$ implies

$$
\left|\frac{z-\lambda_{j}}{z+\lambda_{j}}\right| \geq \frac{2-\lambda_{j}}{2+\lambda_{j}}=\frac{1-\frac{1}{2} \lambda_{j}}{1+\frac{1}{2} \lambda_{j}}, \quad z \in \Gamma .
$$


So, for $z \in \Gamma$,

$$
\left|B_{n}(z)\right| \geq \prod_{j=1}^{n} \frac{1-\frac{1}{2} \lambda_{j}}{1+\frac{1}{2} \lambda_{j}}=\frac{1-\frac{1}{2}}{1+\frac{1}{2}}=\frac{1}{3},
$$

where the inequality

$$
\begin{aligned}
\frac{1-x}{1+x} \frac{1-y}{1+y} & =\frac{1-(x+y)}{1+(x+y)}+\frac{2 x y(x+y)}{(1+x)(1+y)(1+(x+y))} \\
& \geq \frac{1-(x+y)}{1+(x+y)}, \quad x, y \geq 0,
\end{aligned}
$$

was used. We will examine $T_{n, k} \in E\left(\Lambda_{n-1}\right)$ defined by

$$
T_{n, k}(t):=T_{n}^{(k)}(z)=\frac{1}{2 \pi i} \int_{\Gamma} \frac{(-z)^{k} \exp (-z t)}{B_{n}(z)} d z .
$$

Note that the circle $\Gamma$ can be parametrized as

$$
\Gamma:=\{-\exp (i u)+1, u \in[-\pi, \pi)\},
$$

where for $z=-\exp (i u)+1, u \in[-\pi, \pi)$, we have

$$
|z|=|-\exp (i u)+1| \leq|u|
$$

and

$$
|\exp (-z t)| \leq \exp (\operatorname{Re}(-z t))=\exp ((-1+\cos u) t) \leq \exp \left(-\frac{t u^{2}}{12}\right) .
$$

Using the above inequalities together with (3.2), we obtain that

$$
\begin{aligned}
\left|T_{n, k}(t)\right| & \leq\left|\frac{1}{2 \pi i} \int_{-\pi}^{\pi} \frac{(-(-\exp (i u)+1))^{k} \exp (-(-\exp (i u)+1) t)}{B_{n}(-\exp (i u)+1)} i \exp (i u) d u\right| \\
& \leq \frac{1}{2 \pi} \int_{-\pi}^{\pi} \frac{|-\exp (i u)+1|^{k}|\exp (-(-\exp (i u)+1) t)|}{\left|B_{n}(-\exp (i u)+1)\right|}|\exp (i u)| d u \\
& \leq \frac{3}{2 \pi} \int_{-\pi}^{\pi}|u|^{k} \exp \left(-\frac{t u^{2}}{12}\right) d u \\
& \leq \frac{3}{2 \pi} \int_{0}^{2 \pi}\left(\frac{u \sqrt{t}}{\sqrt{12}}\right)^{k} \exp \left(-\left(\frac{u \sqrt{t}}{\sqrt{12}}\right)^{2}\right) \frac{\sqrt{t}}{\sqrt{12}}\left(\frac{\sqrt{12}}{\sqrt{t}}\right)^{k+1} d u \\
& \leq \frac{3}{2 \pi}\left(\int_{0}^{\infty} v^{k} \exp \left(-v^{2}\right) d v\right)\left(\frac{\sqrt{12}}{\sqrt{t}}\right)^{k+1} \\
& \leq c(k) 12^{(k+1) / 2} t^{-(k+1) / 2}
\end{aligned}
$$

with a constant $c(k)$ depending only on $k$. Also,

$$
\begin{aligned}
\left|T_{n, k}(t)\right| & \leq\left|\frac{1}{2 \pi i} \int_{-\pi}^{\pi} \frac{(-(-\exp (i u)+1))^{k} \exp (-(-\exp (i u)+1) t)}{B_{n}(-\exp (i u)+1)} i \exp (i u) d u\right| \\
& \leq \frac{1}{2 \pi} \int_{-\pi}^{\pi} \frac{|-\exp (i u)+1|^{k}|\exp (-(-\exp (i u)+1) t)|}{\left|B_{n}(-\exp (i u)+1)\right|}|\exp (i u)| d u \\
& \leq \frac{3}{2 \pi} 2 \pi 2^{k} \leq 3 \cdot 2^{k} .
\end{aligned}
$$


So with $k:=\lfloor 4 / q\rfloor$ we have

$$
\begin{aligned}
\left\|T_{n, k}\right\|_{L_{q}[0, \infty)} & \leq\left(\int_{1}^{\infty}\left|T_{n, k}(t)\right|^{q} d t+\int_{0}^{1}\left|T_{n, k}(t)\right|^{q} d t\right)^{1 / q} \\
& \left(\int_{1}^{\infty} c(k) 12^{(k+1) q / 2} t^{-(k+1) q / 2} d t+3^{q} \cdot 2^{k q}\right)^{1 / q} \\
& \leq\left(c(k) 12^{(\lfloor 4 / q\rfloor+1) / 2} \int_{1}^{\infty} t^{-2} d t+3^{q} \cdot 2^{4}\right)^{1 / q} \leq C(q)<\infty
\end{aligned}
$$

with a constant $C(q)$ depending only on $q$, and

$$
\left\|T_{n, k}\right\|_{[0, \infty)} \leq C(\infty)<\infty .
$$

Now observe that

$$
\left|T_{n, k}^{(\mu)}(0)\right|=\frac{1}{2 \pi i} \int_{\Gamma} \frac{(-z)^{k+\mu}}{B_{n}(z)} d z .
$$

Here, for all $z \in \mathbb{C}$ with $|z|>1 \geq \max _{1 \leq j \leq n}\left|\lambda_{j}\right|$, we have

$$
\begin{aligned}
\frac{z^{k+\mu}}{B_{n}(z)} & =z^{k+\mu} \prod_{j=1}^{n} \frac{1+\lambda_{j} / z}{1-\lambda_{j} / z}=z^{k+\mu} \prod_{j=1}^{n}\left(1+2 \sum_{m=1}^{\infty}\left(\frac{\lambda_{j}}{z}\right)^{m}\right) \\
& =z^{k+\mu-1}\left(z+2+\sum_{m=1}^{\infty} \frac{A_{m}}{z^{m}}\right)
\end{aligned}
$$

with a constant $A_{m} \geq 1 / m !$. Therefore

$$
\left|T_{n, k}^{(\mu)}(0)\right| \geq \frac{1}{(k+\mu) !}
$$

Pick a point $y \in[0, \infty)$ so that

$$
\left|T_{n, k}^{(\mu)}(y)\right|=\left\|T_{n, k}^{(\mu)}\right\|_{[0, \infty)} .
$$

Note that $T_{n, k}^{(\mu)} \in E\left(\Lambda_{n-1}\right)$. Combining the upper bound of Theorem 1.1 (Newman's inequality) with the Mean Value Theorem, we obtain that

$$
\left|T_{n, k}^{(\mu)}(t)\right| \geq \frac{1}{2}\left\|T_{n, k}^{(\mu)}\right\|_{[0, \infty)}, \quad t \in I:=\left[y, y+\frac{1}{18}\right] .
$$

Hence (3.5) and $k:=\lfloor 4 / q\rfloor$ imply that

$$
\begin{aligned}
\left\|T_{n, k}^{(\mu)}\right\|_{L_{p}[0, \infty)} & \geq\left(\frac{1}{18}\left(\frac{1}{2}\left\|T_{n, k}^{(\mu)}\right\|_{[0, \infty)}\right)^{p}\right)^{1 / p} \\
& \geq\left(\frac{1}{18}\left(\frac{1}{2} \frac{1}{(k+\mu) !}\right)^{p}\right)^{1 / p} \\
& \geq c(p, q, \mu)>0
\end{aligned}
$$

and

$$
\left\|T_{n, k}^{(\mu)}\right\|_{[0, \infty)} \geq \frac{1}{(k+\mu) !} \geq c(\infty, q, \mu) .
$$

Combining (3.3)-(3.7) finishes the proof. 


\section{REFERENCES}

1. P. B. Borwein and T. Erdélyi, Polynomials and Polynomials Inequalities, Springer-Verlag, New York, 1995. MR 97e:41001

2. P. B. Borwein and T. Erdélyi, The $L_{p}$ version of Newman's inequality for lacunary polynomials, Proc. Amer. Math. Soc. 124 (1996), 101-109. MR 96j:41015

3. T. Erdélyi, Markov- and Bernstein-type inequalities for Müntz polynomials and exponential sums in $L_{p}$, J. Approx. Theory 104 (2000), 142-152. MR 2001c:41014

4. D. J. Newman, Derivative bounds for Müntz polynomials, J. Approx. Theory 18 (1976), 360362. MR 55:3609]

Department of Mathematics, Texas A\&M University, College Station, Texas 77843

E-mail address: terdelyi@math.tamu.edu 